\title{
Bacterial typing systems: the way ahead
}

In the 19th and early 20th century many different species of pathogenic bacteria were described, and sets of key phenotype characters were established to identify members of them. Later, the idea became current that the bacterial species was a group of isolates showing maximal similarity in all ascertainable phenotypic and genotypic characters, often referred to as "overall similarity". However, species may often be subdivided ("typed") on the basis of characters of a single class; (biotyping, serotyping, phage typing, bacteriocin typing), and practical use of this may be made to obtain information about sources and routes of infection. Membership of the same type gives some presumption, but never certainty, of an epidemiological relationship. However, as pointed out by Anderson and Williams, ${ }^{1}$ typing has a more important role in excluding from consideration isolates that differ from the index strain, thus narrowing the field of search for those relevant in the incident under investigation.

Typing may assist us in halting a current outbreak of infection; more often, by contributing to our knowledge of the pattern of spread of infection, it may influence the design of preventive programmes. Occasionally, by showing that a disease is type-associated, it may provide a valuable lead to the pathophysiology of an organism, e.g. nephritogenicity in group-A streptococci and enteropathogenicity in Escherichia coli, but instances of this sort are comparatively rare.

In the process of type identification of bacterial strains, reference schemes based on biological characteristics are established against which field isolates can be compared. Many systems became universally accepted as the reference method for a particular species, e.g. the Kauffmann-White classification of Salmonella spp. and the Lancefield grouping of streptococci. These schemes defined serological types or groups within a species and the frequency of individual groups determined the discriminatory power of the scheme. ${ }^{2}$

It soon became clear that further division could be made within most types if alternative markers such as sensitivity to, or production of, bacteriophages or bacteriocins were used. A hierarchy of type characters evolved in which the serotype usually formed the primary division of isolates as it was often the most reproducible and a clear distinction between types could be made. Methods such as phage typing, which produced patterns that overlapped between isolates, were more suitable as secondary systems. In practice, isolates from a cluster of infections belonging to different primary types are considered to be distinct strains and need not be examined further. Isolates of the same primary type are subjected to further typing by a secondary system. Occasionally, a third method may be necessary to differentiate between similar strains. Nowadays, this is often a molecular method providing some sort of genomic profiling.

Naturally, there is a limit to the degree of subdivision of strains that can be achieved or is desirable, but if major or minor differences can be consistently demonstrated in a pair of isolates indistinguishable by other methods then this must be significant. Reproducibility is the limiting factor influencing the level of subdivision that is useful in defining a strain type among similar isolates.

A typing method should give reproducible results in both the laboratory and the clinical context. The stability of a method may be influenced by many variables, including culture conditions and the structural and genetic integrity of the marker identified by the method. Cultural variation can be controlled but often little can be done about the expression of an antigen epitope or phage-receptor site on the cell surface. Similarly, with genomic profiling methods, a point mutation that does not alter the strain identity may result in one or two band differences between isolates of the same strain. The question of how many differences in pattern or electrophoretic profile are allowable before one is confident that two isolates are distinct remains open.

Williams and Rippon in $1952^{3}$ proposed the first set of "reaction-difference rules" for the interpretation of phage-typing patterns in sets of strains of Staphylococcus aureus. They established different sets of cultures of staphylococci: (a) to act as tests of the method itself; (b) to determine the degree of variability in natural staphylococcal populations in nasal carriers; and (c) to examine the constancy of phage patterns of strains in outbreaks. As a result they proposed that isolates of $S$. aureus that differed by two or more strong phage lytic reactions in simultaneous tests should be regarded as distinct. However, as staphylococcal phages may be grouped by their lytic spectra, a single strong reaction difference between phages in different lytic groups usually indicated a distinct strain. The comparison of patterns obtained on different days was more difficult and although the same rules were applied, the results were reported with "less confidence". Unfortunately, this type of critical appraisal of a method has gone out of fashion, but it is perhaps because of the establishment of such ground rules that staphylococcal phage typing has remained a useful and widely applied method whilst many of its infant rivals have not. 
There is no right way to develop or evaluate a typing system, but any phenotypic property used as a strain marker should allow geographically and epidemiologically distinct strains to be distinguished. For the development or evaluation of a typing system, a comprehensive panel of reference strains-representatives from past outbreaks and, if possible, a few "natural" strains not associated with disease-is required. A second panel of isolates is recommended comprising, as appropriate: colonial variants of the same strain; pairs of isolates from the same and different sites of the same patient; multiple colonies from primary platings of specimens; and antibiotic sensitive and resistant sets from the same patient. Thus, the epidemiology of the infection exerts the most influence on the typing system under development.

Many of the newer molecular typing techniques generate bands in a gel or on a membrane that are then compared for similarity. The methods are comparative and do not usually define a type against a reference band profile. Various attempts have been made to apply computerised scanning to the analysis of patterns but these have not been adopted widely because of cost and the evolutionary state of the software. As a result, most workers compare isolates pair-wise and present strain relationships in rank order of similarity, e.g. as a dendrogram.

The literature is replete with accounts of the use of various molecular techniques to type collections of isolates of an increasing number of bacterial species. Some researchers seem intent on applying their chosen technique to each member of the Bacteriological Code in turn with little or no further development and evolution. This fails to take the subject forward in a way that is helpful to prospective users.

So, where is typing going? The answer is not clear but the newer methods are providing significant advances

\section{References}

1. Anderson ES, Williams REO. Bacteriophage typing of enteric pathogens and staphylococci and its use in epidemiology. $J$ Clin Pathol 1956; 9: 94-127.

2. Hunter PR, Gaston MA. Numerical index of the discriminatory ability of typing systems: an application of Simpson's index of diversity. J Clin Microbiol 1988; 26: 2465-2466.

3. Williams REO, Rippon JE. Bacteriophage typing of Staphylococcus aureus. J Hyg 1952; 50: 320-353.

4. Selander RK, Beltram P, Smith NH et al. Genetic population structure, clonal phylogeny and pathogenicity of Salmonella paratyphi B. Infect Immun 1990; 58: 1891-1901.

5. Crowe BA, Wall RA, Kusecek B et al. Clonal and variable properties of Neisseria meningitidis isolated from cases and carriers during and after an epidemic in The Gambia, West Africa. J Infect Dis 1989; 159: 686-700.

6. Musser JM, Kroll JS, Granoff DM et al. Global genetic in our understanding of bacterial populations: evidence of the genetic diversity of strains within species involved in outbreaks; ${ }^{4,5}$ clues to the clonal or common ancestry of epidemic strains world-wide, sometimes associated with antibiotic resistance $;{ }^{6,7}$ and the inter-relationship between genotype and phylogenetic origin as indicated by rRNA genes. ${ }^{8}$ The genotyping techniques will, no doubt, be refined and simplified, and the ability to design primers and amplify DNA will be used to increase specificity and sensitivity respectively. ${ }^{9,10}$

Comparative typing and fingerprinting of isolates within incidents of infection will become increasingly common as the technology becomes more readily available and easier to use. Some clinical laboratories may be tempted to do their own typing in response to infection control needs. This should not be encouraged as it may lead to the generation of locally relevant data which cannot be integrated with the regional and national picture, the problem being of standardisation of results of the new techniques from laboratories using slightly different protocols. Furthermore, it is doubtful whether results would be more rapidly available since the method of choice may have to be first set up and standardised.

The traditional phenotype systems that have stood the test of time should prevail because they are simple and because they do often accurately reflect the genotype of strains. The reference laboratory will, moreover, continue to be needed to monitor regional, national and international movement of strains and to develop and validate type identification systems with reference to established "gold standard" schemes.

T. L. PitT

Laboratory of Hospital Infection, Central Public Health Laboratory, 61 Colindale Avenue, London NW9 5HT.

structure and molecular epidemiology of encapsulated Haemophilus influenzae. Rev Infect Dis 1990; 12: 75-111.

7. Pitt TL, Livermore DM, Pitcher D, Vatopoulos AC, Legakis NJ. Multiresistant serotype $\mathrm{O} 12$ Pseudomonas aeruginosa evidence for a common strain in Europe. Epidemiol Infect $1989 ; 103$ : 565-576.

8. Fry NK, Warwick S, Saunders NA, Embley TM. The use of $16 \mathrm{~S}$ ribosomal RNA analyses to investigate the phylogeny of the family Legionellaceae. J Gen Microbiol 1991; 137: 1215-1222

9. Caetano-Anollés G, Bassam BJ, Gresshoff PM. DNA amplification fingerprinting using very short arbitrary oligonucleotide primers. Biotechnology 1991; 9: 553-557.

10. Kostman JR, Edlind TD, LiPuma JJ, Stull TL. Molecular epidemiology of Pseudomonas cepacia determined by polymerase chain reaction ribotyping. J Clin Microbiol 1992; 30: 2084-2087 